\title{
Fracture Resistance of Ceramic Laminate Veneers Bonded to Teeth with Class V Composite Fillings after Cyclic Loading
}

\author{
Leyla Sadighpour (D), ${ }^{1}$ Farideh Geramipanah ${ }^{(D)}{ }^{2}$ Vanya Rasaei, ${ }^{1}$ \\ and Mohammad J. Kharazi Fard ${ }^{3}$ \\ ${ }^{1}$ Department of Prosthodontics, Faculty of Dentistry, Tehran University of Medical Sciences, Tehran, Iran \\ ${ }^{2}$ Dental Implant Research Center, Faculty of Dentistry, Tehran University of Medical Sciences, Tehran, Iran \\ ${ }^{3}$ Dental Research Center, Faculty of Dentistry, Tehran University of Medical Sciences, Tehran, Iran \\ Correspondence should be addressed to Farideh Geramipanah; geramipa@tums.ac.ir
}

Received 9 November 2017; Revised 14 March 2018; Accepted 26 March 2018; Published 17 April 2018

Academic Editor: Ali I. Abdalla

Copyright (c) 2018 Leyla Sadighpour et al. This is an open access article distributed under the Creative Commons Attribution License, which permits unrestricted use, distribution, and reproduction in any medium, provided the original work is properly cited.

\begin{abstract}
Purpose. Porcelain laminate veneers (PLVs) are sometimes required to be used for teeth with composite fillings. This study examined the fracture strength of PLVs bonded to the teeth restored with different sizes of class V composite fillings. Materials and Methods. Thirty-six maxillary central incisors were divided into three groups $(n=12)$ : intact teeth (control) and teeth with class $\mathrm{V}$ composite fillings of one-third or two-thirds of the crown height (small or large group, resp.). PLVs were made by using IPS e.max and bonded with a resin cement (RelyX Unicem). Fracture resistance $(N)$ was measured after cyclic loading $\left(1 \times 10^{6} \mathrm{cycles}, 1.2 \mathrm{~Hz}\right)$. For statistical analyses, one-way ANOVA and Tukey test were used $(\alpha=0.05)$. Results. There was a significant difference between the mean failure loads of the test groups $(P=0.004)$, with the Tukey-HSD test showing lower failure loads in the large-composite group compared to the control $(P=0.02)$ or small group $(P=0.05)$. The control and small-composite groups achieved comparable results $(P>0.05)$. Conclusions. Failure loads of PLVs bonded to intact teeth and to teeth with small class V composite fillings were not significantly different. However, extensive composite fillings could compromise the bonding of PLVs.
\end{abstract}

\section{Introduction}

Porcelain laminate veneers (PLVs) represent an excellent treatment option for restoring aesthetics while preserving the integrity of the remaining tooth structure. Its clinical acceptance is mainly due to minimal preparation, successful clinical outcomes, and high patient satisfaction [1]. The estimated survival rate of PLVs has been reported to be $94.4 \%$ after five years and $93.8 \%$ after 10 years [2]. The most frequent causes of failure, including microleakage, debonding, and fracture, are related to the quality of the bonding surface and the choice of the luting agent $[3,4]$. Because the retention of PLVs relies solely on the bond to the tooth structure, the availability of sufficient enamel has been considered as a critical factor for its long-term success [5]. For a predictable result, it is recommended that at least $50 \%$ of the labial enamel or intraenamel margins be present $[6,7]$. The amount of enamel may be compromised by several factors, such as fracture, wear, and preparation of various types of restorations, including composite fillings [8-10]. Even standard preparations for PLVs could result in serious enamel damage. Various preparation techniques could compromise $30 \%$ to $50 \%$ of the labial surface enamel, leading to dentin exposure $[11,12]$.

Patients commonly seek esthetic treatment for discoloured or aged composite fillings $[7,13]$. One study on the clinical performance of PLVs showed that $70 \%$ of patients had previous composite fillings [2]. Under such circumstances, some clinicians believe that the preparation margins should be extended to sound enamel, far to the interproximal or even to the lingual enamel, to create intraenamel margins $[7,8]$. Findings from several clinical studies support this approach. For example, higher failure rates were observed when laminate veneers were bonded to large-composite fillings [2, 3]. A reliable bonding could be achieved with greater quantities of enamel surface and margins. 
However, this process compromises excess dental hard tissue $[13,14]$.

An alternative approach is to bond PLV onto an acceptable composite filling $[15,16]$. Gurel et al. [12] found no relation between the existing restorations and PLV failure. Similarly, Gresnigt et al. [17] found no significant difference in the 40-month clinical performances of PLVs bonded to teeth with and without existing composite fillings. Moreover, the overall survival rate remained high, at $94.6 \%$. Although the available literature suggests that the size, age, and location of composite fillings are among the factors that may influence the failure rate of PLVs bonded to existing restorations, these factors have not been clearly identified over literature. Quantifying the amount of enamel that can be replaced by composite fillings without affecting the fracture resistance may improve our clinical decisionmaking. Few investigations have been conducted with a focus on the performance of PLVs when bonded to composite fillings with defined dimensions [16-18]. In an in vitro study, Sadighpour et al. [18] found that fracture resistance of PLVs bonded to intact teeth or teeth with a classic class III composite filling was similar. In addition, fracture resistance decreased and microleakage of PLVs increased when the composite fillings were removed and replaced by veneers. Class V composite fillings could differ from class III fillings in location, size, and stress patterns [19]. Furthermore, a typical class $\mathrm{V}$ cavity could compromise a greater amount of labial enamel than either a class III or IV cavity that has not been investigated.

In the light of this background, the aim of the present study was to examine the fracture strength of PLVs bonded to teeth restored with different sizes of class $\mathrm{V}$ composite fillings. The null hypothesis was that the fracture strength of teeth restored with PLVs would not be affected by the presence of class $\mathrm{V}$ composite fillings of different sizes.

\section{Materials and Method}

The sample for this study included 36 human maxillary central incisors that had been extracted from adults within the past three months due to periodontal problems. All patients provided informed consent for extraction and subsequent use of their teeth for experimental study, according to the protocol of the Clinical Research Ethics Board at Tehran University of Medical Sciences. Only teeth with no caries, cracks, or excessive wear were included in the study.

Teeth were cleaned of tissue tags and debris by using a hand instrument and were stored in $1 \%$ chloramine for two weeks and then in distilled water until use. The teeth fell into three groups based on the labial surface area $(65 \pm 5 \mathrm{~mm}$, $75 \pm 5 \mathrm{~mm}$, and $85 \pm 5 \mathrm{~mm}$ ).

Teeth from each size group were randomly assigned to three groups: control (NC) (intact with no composite fillings), small-composite (SC), and large-composite (LC) $(n=12)$.

In the $\mathrm{NC}$ group, the incisal edges were reduced by $1 \mathrm{~mm}$. Three orientation grooves were made on the labial surface by a $0.3 \mathrm{~mm}$ cutting-depth diamond bur (868B.314.018, Komet

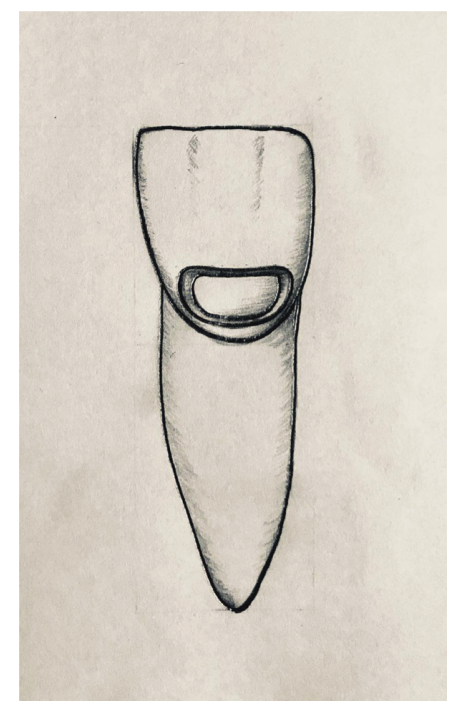

(a)

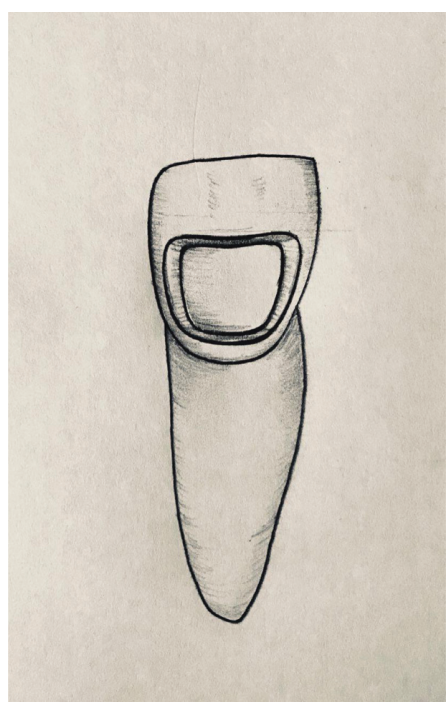

(b)

Figure 1: (a) Representative of a small class V cavity (b) and large class $\mathrm{V}$ cavity.

Dental, Lemgo, Germany) and were joined together by a round-end diamond bur (868.018, Komet Dental). Cervical finish lines were ended $1 \mathrm{~mm}$ above the cementoenamel junction (CEJ) to ensure intraenamel margins. Proximally, the reduction was extended by $1 \mathrm{~mm}$ toward the lingual surface. All line angles were rounded, and the margins were finished.

In SC and LC groups, a class V cavity at one-third or two-thirds of the crown height, respectively, was prepared for composite filling. Each cavity was $1 \mathrm{~mm}$ short of the proximal finish line and $2 \mathrm{~mm}$ short of the CEJ, Figure 1 . Autopolymerised acrylic resin (GC Pattern Resin, GC America Inc., Alsip, IL, USA) was used in one cavity each from the SC and LC groups to fabricate a template for the remaining teeth, Figure 2. Cavities were etched for $15 \mathrm{~s}$ with $38 \%$ phosphoric acid (Etching Gel, Faghihi Dental Co., Tehran, Iran), rinsed for $15 \mathrm{~s}$, and then gently air dried. 


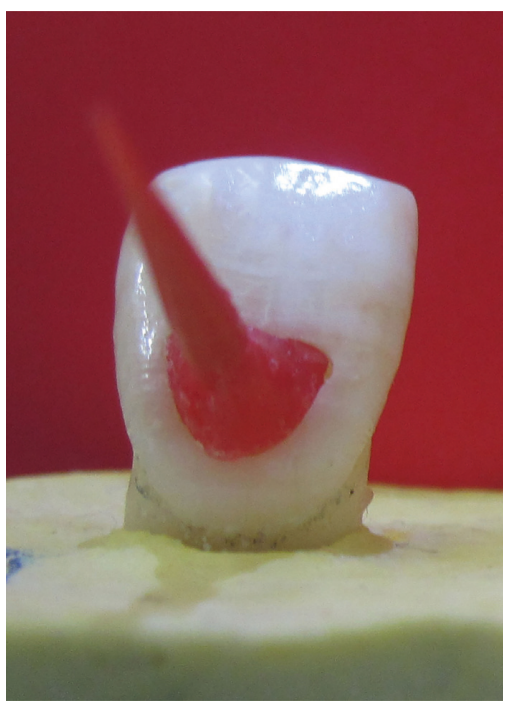

FIGURE 2: Resin template for guiding the cavity preparation.

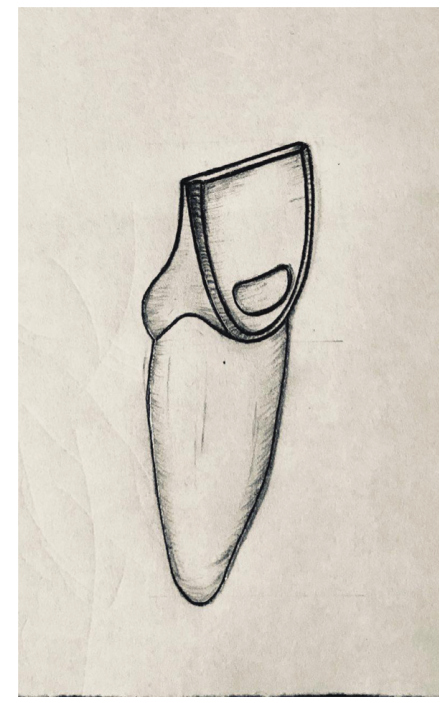

FIGURE 3: Schematic representation of final preparation.

An etch-and-rinse dentin bonding agent (Single Bond, 3M ESPE, MN, USA) was applied for 30s and light polymerised (Bluephase 16i, Ivoclar/Vivadent, Schaan, Liechtenstein) with a $5 \mathrm{~s}$ burst at $1100 \mathrm{~mW} / \mathrm{cm}^{2}$. Cavities were restored by using a universal microhybrid composite resin (Filtek Z250, $3 \mathrm{M}$ ESPE), which was inserted in two layers and light polymerised with an LED light unit (Bluphase 16i). Teeth were prepared for PLV in a similar way as in the NC group, Figure 3. Specimens were stored in water at room temperature of $25^{\circ} \mathrm{C}$ for three weeks before receiving veneers. A custom tray was fabricated with autopolymerising acrylic resin (Tray Material, Major, Moncalieri, Italy) for each specimen. Impressions were made by a polyvinyl siloxane material (Monopren, Kettenbach GmbH, Eschenburg, Germany) and poured with type IV dental stones (Fuji Rock, GC Corp., Tokyo, Japan). PLVs were fabricated with a lithium disilicate ceramic (IPS e.max Press, Ivoclar/Vivadent) using the lostwax technique. Wax patterns were fabricated at a uniform thickness of $0.7 \mathrm{~mm}$, sprued with four patterns per cylinder, invested with a phosphate-bound investment (IPS Pres/Vest, Vivadent/Ivoclar), and burned out in a conventional porcelain-firing furnace (Vaccumat 300, Vita, Zahnfabrik $\mathrm{H}$. Rauter $\mathrm{GmbH}$, Bad Sackingen, Germany) at $850^{\circ} \mathrm{C}$. Lowtranslucency ceramic ingots in shade A2 were pressed in a Programat furnace (Ivoclar/Vivadent) at $1050^{\circ} \mathrm{C}$. Molds were divested by using $50 \mu \mathrm{m}$ aluminium oxide air particles at $0.4 \mathrm{MPa}$ and a distance of $10 \mathrm{~mm}$ for $5 \mathrm{~s}$ and then cleaned in an ultrasonic cleaner (Prosonic 300, Sultan Health Care, Englewood, NJ, USA).

PLVs on teeth were investigated for any cracks or flaws under 5x magnification, and the fit was examined on the corresponding dies. PLVs were glazed (IPS e.max Ceram Glaze, Vivadent/Ivoclar) in a conventional porcelain-firing furnace (Vacumat 300 ) at $750^{\circ} \mathrm{C}$. PLVs were etched with $9.5 \%$ hydrofluoric acid (Porcelain Etchant, Bisco Inc., Schaumburg, IL, USA) for 90s, cleaned under water for $30 \mathrm{~s}$ and then silanized (Monobond S, Vivadent/Ivoclar). Enamel surfaces and margins were etched by $37 \%$ phosphoric acid (Etching Gel) for $15 \mathrm{~s}$ and washed for $15 \mathrm{~s}$. A self-adhesive resin cement (RelyX Unicem, Automix, 3M/ESPE, St Paul, MN, USA) was applied on the bonding surface of veneers and then seated on the tooth surface and held in place with finger pressure for $10 \mathrm{~s}$. The excess cement was removed by a scalpel blade. Light polymerisation (Bluephase 16i, Vivadent/Ivoclar) was performed for $10 \mathrm{~s}$. The specimens were stored for $24 \mathrm{~h}$ in water at room temperature before being subjected to thermal cycling (2000 thermal cycles between $5^{\circ} \mathrm{C}$ and $55^{\circ} \mathrm{C}$, with a dwell time of $30 \mathrm{~s}$ in each bath and a transfer time of $10 \mathrm{~s}$ ). The periodontal ligament was simulated with polyether elastomer (Impergum F, 3M/ESPE) on the roots of the teeth before being mounted on a chewing simulator. Specimens were mounted at $135^{\circ}$ with the contact point on the cingulum. To simulate three years of service, specimens were subjected to $1.2 \times 10^{6}$ cycles of $50 \mathrm{~N}$ loads at a frequency of $2 \mathrm{~Hz}$.

Fracture resistance was measured by applying a static load to the specimens until fracture using a universal testing machine (Zwick Roell Z050, Ulm, Germany). A compressive load was applied at $1 \mathrm{~mm} / \mathrm{min}$ to the incisal edge of the specimens with a stainless-steel sphere, $8 \mathrm{~mm}$ in diameter, and a thin sheet in between the contact points. The fracture load was automatically recorded as the fracture resistance of each PLV.

The failure mode was examined with a stereomicroscope (Zeiss OPM1, Carl Zeiss, Oberkochen, Germany) at a magnification of $\times 20$. The failure mode was categorised as adhesive (failure between the tooth and laminate), cohesive (failure within the laminate or tooth), or mixed. The homogeneity of variances and normal distribution of data were verified by Levene's test and the Kolmogorov-Smirnov test, respectively. Then, the mean values of fracture resistance were statistically analysed by one-way ANOVA and TukeyHSD post hoc using SPSS (SPSS Inc., Chicago, IL, USA) at a significance level of $95 \%(\alpha=0.05)$.

\section{Results}

During cyclic loading, one specimen each in the NC and SC groups and two specimens in the LC group were lost due to 
TABle 1: The descriptive data for failure load values of test groups.

\begin{tabular}{lcccccc}
\hline Test group & Number & Mean $(N)$ & Standard deviation $(N)$ & Min. $(N)$ & Max. $(N)$ & $\begin{array}{c}\text { 95\% confidence interval } \\
\text { Lower bound }\end{array}$ \\
& & & & & & Upper bound \\
\hline NC & 11 & $364.28^{\mathrm{a}}$ & 114.88 & 219.30 & 616.50 & -296.46 \\
SC & 11 & $324.57^{\mathrm{a}}$ & 89.25 & 220.10 & 471.4 & -271.88 \\
LC & 10 & $207.26^{\mathrm{b}}$ & 90.62 & 110.10 & 369.00 & -151.05 \\
\hline
\end{tabular}

Items in the rows with dissimilar letters are significantly different at $95 \%$ level of confidence; ${ }^{a}$ values are nonsignificantly different at $P=0.81$; ${ }^{\mathrm{b}}$ value is significantly different at $P=0.004$.

TABLE 2: Failure modes of broken specimens.

\begin{tabular}{lcccc}
\hline Group & $\begin{array}{c}\text { Cohesive } \\
\text { fracture } \\
\text { in tooth }\end{array}$ & $\begin{array}{c}\text { Cohesive } \\
\text { fracture in } \\
\text { laminate }\end{array}$ & Adhesive & $\begin{array}{c}\text { Mixed } \\
\text { cohesive and } \\
\text { adhesive }\end{array}$ \\
\hline Control & 5 & 1 & 0 & 5 \\
SC & 4 & 0 & 3 & 4 \\
LC & 1 & 0 & 5 & 4 \\
Total & 10 & 1 & 8 & 13 \\
\hline
\end{tabular}

fracture. Fracture value for the lost specimens was set to zero.

The descriptive data of fracture resistance are summarised in Table 1 . The mean failure loads were significantly different among the test groups $(P=0.004$ by oneway ANOVA). Tukey-HSD revealed that the failure load in the LC group was lower than the failure load in the $\mathrm{NC}$ or SC group ( $P=0.02$ and 0.05 , resp., by Tukey's test). Adhesive failure mode was dominant in the LC group, and cohesive failure occurred more often in the NC and SC groups (Table 2).

\section{Discussion}

The mean fracture resistance of the PLV-restored teeth with class $\mathrm{V}$ composite fillings was examined after cyclic loading. No difference in fracture resistance was found between teeth with composite filling to one-third of the crown height (small-composite group) compared to teeth with an intact labial surface (control). However, the mean fracture resistance was significantly lower in the large-composite group compared to the small-composite or control group. Therefore, the null hypothesis should be partially accepted.

In this study, teeth with $33 \%$ of the original enamel remaining (one-third) on the labial surface showed the lowest fracture strength among the groups. Gurel et al. [12] have also emphasised on the role of the remaining enamel on the failure rates of PLVs. They found that PLVs bonded to the dentin had 10 times greater risk of failure than PLVs bonded to the enamel. In contrast, the presence of composite fillings was not correlated with an increased risk of failure, and PLVs bonded to composite fillings had outcomes that were more favourable. However, excessive dentinal exposure, dentinal margins, and the size of composite fillings were not quantified by the authors. Therefore, a direct comparison could not be performed with our study. Contrary to the findings of our study, Gresnigt et al. [17] found that the size of composite fillings did not affect the survival rate of PLVs over a period of 40 months. The size categories in their study were close to those in the small- and largecomposite groups of our study. The chairside application of surface silica coating on the resin composite surface before PLV bonding may explain the discrepancy between the studies. Furthermore, the overall follow-up time of their study was short, and most failures occur after years of service $[3,7]$. In the present study, fatigue ageing was performed by applying cyclic loading in an attempt to simulate a three-year clinical service.

The luting agent is another contributing factor to the longevity of adhesive restorations [4]. We used a selfadhesive cement, which allows bonding to a range of substrates without pretreatment. The multiple steps of etching, rinsing, and adhesive application are omitted with selfadhesive cement, thereby overcoming the technique and handling errors [20]. Moreover, the setting mechanism of RelyX Unicem self-adhesive cement permits setting with low shrinkage [21]. Contraction during the setting of the resin cement could produce stress within the cement layer, which might induce debonding or cracks in the ceramic over time [22]. However, self-adhesive cement also has some disadvantages, such as lower bond strengths compared to the cement that utilises the etch-and-rinse bonding system. [23]. Nevertheless, in the present study, it was attempted to simulate the worst-case scenario, in that the bond of resin cement to tooth structure was low. Furthermore, for ceramic surface treatment, a protocol was followed that provided a strong bond to ensure no failure from ceramic/cement interface. Therefore, veneers were etched with a 9.5\% $\mathrm{HF}$ for 90 seconds that has been showed to increase the bond strength of lithium disilicate ceramic to resin cements [24-26].

Regardless of the polymerisation mode, the bonding of resin cement to another resin occurs through a mechanism of free-radical polymerisation and covalent bonding to the organic matrix. However, when a PLV is indicated on a tooth with an old composite filling, the bonding between the resin cement and composite may be compromised due to reduced unreacted monomer because of several degradation processes such as water sorption/solubility, $\mathrm{pH}$ action, and mechanical breakdown [27]. The greatest amount of free monomer is present on the surface of a composite filling during the first $24 \mathrm{~h}$ after polymerisation and decreases dramatically after one month [15]. In the present study, PLVs were bonded after storage in water for three weeks. Accordingly, minor or no free monomer could be expected on the surface of composite fillings. Unicem contains special methacrylate monomers with bonded phosphoric acid groups with at least two carbon double bonds. It is suggested 
that the acidic functional monomer in Unicem may ease penetration into the polymeric structure of a composite resin and mediate the acid-base setting reaction with the organic fillers available in the polymer matrix [22]. Simultaneously, radical polymerisation of methacrylate monomers through reactive carbon double bonds promotes copolymerisation with another polymer material [21].

Various surface treatments have been incorporated to improve the bond of new composite resin with an old one including sillicoating, chemical and mechanical roughening, and application of adhesive layers [28, 29]. However, the findings were not consistent. Furthermore, it was shown that the result is highly material-dependent [30]. Therefore, in the present study, the surface of composite fillings was ground using diamond burs, and no further treatment was applied to avoid extra confounding parameters. Surface treatments (e.g., airborne-particle abrasion, acid etching, silanization, and application of a lowviscosity adhesive) might improve the bonding of resin cement to old composites; therewith more favourable outcomes may be expected that could be investigated in future studies.

In the present study, we designed the intraenamel margins to simplify the research parameters. In the clinic, dentinal exposure is inevitable in most PLV preparations, particularly in the cervical area where the enamel is thin $(<0.5 \mathrm{~mm})[1,12]$. Thus, the effect of the dentinal margin in class $\mathrm{V}$ composite fillings should be tested in future studies.

In our study, laminate fracture was rare, and tooth fracture and mixed failure were the two most occurring failure modes in the NC and SC groups (Table 2). It could be argued that in teeth with larger-composite filling (LC), more enamel has been removed, and a decrease in the elastic modulus of the tooth could result in more distortion under loading and an increased rate of debonding. Early debonding may save the LC group from fracture and increase the frequency of adhesive and mixed failure [4, 31]. On the other hand, in the NC and SC groups, adhesive failure was rare. Higher fracture resistance in these groups may imply that the bond strength was high enough to withstand cyclic loading and eventually failure would occur cohesively within the cement layer and/or the tooth itself $[4,13,14]$.

This was an in vitro study, and the findings cannot be directly extrapolated to the clinical situation. Nonetheless, several conditions were applied to fit the clinical situation. Thermal cycling and load cycles were applied to simulate certain conditions of real-life restorations. Natural teeth were selected because they have unique properties, such as elasticity, enamel, and dentin bonding properties, as well as a complex geometry of preparation, which may influence the findings of the study. However, we faced obstacles in terms of tooth availability and the standardisation of the tooth size and age. Nevertheless, the results should be regarded with caution as a perfect simulation was not possible. Finally, only one cement type was used in the present study. Further studies involving diverse types of resin cement and composite surface treatments should be performed.

\section{Conclusion}

Within the limitation of the present study, significantly stronger bonds were achieved in the control and smallcomposite groups compared to the large-composite group. This result was supported by the prevalence of cohesive failures in the control and small-composite groups. Adhesive failure was predominant in the large-composite group, confirming the weaker bond strength in this group.

\section{Conflicts of Interest}

The authors declare that they have no conflicts of interest.

\section{Acknowledgments}

This research has been supported by the grant no. 21162 from Tehran University of Medical Sciences. The authors are also grateful to Mr. Hassanzadeh for his technical assistance in PLV fabrication.

\section{References}

[1] E. Ozturk and S. Bolay, "Survival of porcelain laminate veneers with different degrees of dentin exposure: 2-year clinical results," Journal of Adhesive Dentistry, vol. 16, no. 5, pp. 481-489, 2014.

[2] M. Peumans, J. De Munck, S. Fieuws, P. Lambrechts, G. Vanherle, and B. Van Meerbeek, "A prospective ten-year clinical trial of porcelain veneers," Journal of Adhesive Dentistry, vol. 6, no. 1, pp. 65-76, 2004.

[3] U. S. Beier, I. Kapferer, D. Burtscher, and H. Dumfahrt, "Clinical performance of porcelain laminate veneers for up to 20 years," International Journal of Prosthodontics, vol. 25, no. 1, pp. 79-85, 2012.

[4] H. S. Cotert, M. Dundar, and B. Ozturk, "The effect of various preparation design on the survival of porcelain laminate veneers," Journal of Adhesive Dentistry, vol. 11, no. 5, pp. 405-411, 2009.

[5] G. Ibarra, G. H. Johnson, W. Geurtsen, and M. A. Vargas, "Microleakage of porcelain veneer restorations bonded to enamel and dentin with a new self-adhesive resin based dental cement," Dental Materials, vol. 23, no. 2, pp. 218-225, 2007.

[6] F. J. T. Burke, "Survival rates for porcelain laminate veneers with special reference to the effect of preparation in dentine: a literature review," Journal of Esthetic and Restorative Dentistry, vol. 22, no. 4, pp. 257-265, 2012.

[7] M. Fradeani, M. Redemagni, and M. Corrado, "Porcelain laminate veneers: 6- to 12-year clinical evaluation-a retrospective study," International Journal of Periodontics and Restorative Dentistry, vol. 25, no. 1, pp. 9-17, 2005.

[8] A. C. Kasaz, C. E. Pena, R. S. de Alexandre et al., "Effects of a peripheral enamel margin on the long-term bond strength and nano leakage of a composite/dentin interface produced by self-adhesive and conventional resin cements," Journal of Adhesive Dentistry, vol. 14, no. 3, pp. 251-263, 2012.

[9] K. K. Schmidt, Y. Chiayabutr, K. M. Phillips, and J. C. Kois, "Influence of preparation design and existing condition of tooth structure on load to failure of ceramic laminate veneers," Journal of Prosthetic Dentistry, vol. 105, no. 6, pp. 374-382, 2011.

[10] G. Batalocco, H. Lee, C. Ercoli, C. Feng, and H. Malmstrom, "Fracture resistance of composite resin restorations and 
porcelain veneers in relation to residual tooth structure in fractured incisors," Dental Traumatology, vol. 28, no. 1, pp. 75-80, 2012.

[11] G. P. Cherukara, G. R. Davis, K. G. Seymour, L. Zou, and D. Y. Samarawichrama, "Dentin exposure in tooth preparation for porcelain veneers: a pilot study," Journal of Prosthetic Dentistry, vol. 94, no. 5, pp. 414-420, 2005.

[12] G. Gurel, N. Sesma, M. A. Calamita, C. Coachman, and S. Morimoto, "Influence of enamel preservation on failure rates of porcelain laminate veneers," International Journal of Periodontics and Restorative Dentistry, vol. 33, no. 1, pp. 3139, 2013.

[13] Y. P. Chun, C. Raffelt, H. Pfeiffer et al., "Restoring strength of incisors with veneers and full ceramic crowns," Journal of Adhesive Dentistry, vol. 12, no. 1, pp. 45-54, 2010.

[14] M. Ozcan and A. Mese, "Fracture strength of indirect resin composite laminates to teeth with existing restorations: an evaluation of conditioning protocols," Journal of Adhesive Dentistry, vol. 11, no. 5, pp. 391-397, 2009.

[15] C. Maneenut, R. Sakoolnamarka, and J. M. J. Tyas, “The repair potential of resin composite materials," Dental Materials, vol. 27, no. 2, pp. e20-e27, 2011.

[16] M. M. Grestnigt, M. Ozcan, W. Kalk, and G. Galhano, "Effect of static and cyclic loading on ceramic laminate veneers adhered to teeth with and without aged composite restorations," Journal of Adhesive Dentistry, vol. 13, no. 6, pp. 567$579,2011$.

[17] M. M. Gresnigt, W. Kalk, and M. Ozcan, "Clinical longevity of ceramic laminate veneers bonded to teeth with and without existing composite restorations up to 40 months," Clinical Oral Investigations, vol. 17, no. 3, pp. 823-832, 2012.

[18] L. Sadighpour, F. Geramipanah, S. Allahyari, B. Fallahi Sichani, and M. J. Kharazi Fard, "In vitro evaluation of the fracture resistance and microleakage of porcelain laminate veneers bonded to teeth with composite fillings after cyclic loading," Journal of Advanced Prosthodontics, vol. 6, no. 4, pp. 278-294, 2014.

[19] J. S. Rees and P. H. Jacobsen, "The effect of interfacial failure around a class $\mathrm{V}$ composite restoration analyzed by the finite element method," Journal of Oral Rehabilitation, vol. 27, no. 2, pp. 111-116, 2000.

[20] E. Ozturk, S. Bolay, R. Hickel, and N. Ilie, "Shear bond strength of porcelain laminate veneers to enamel, dentin and enamel-dentin complex bonded with different adhesive luting systems," Journal of Dentistry, vol. 41, no. 2, pp. 97-105, 2013.

[21] J. L. Ferracane, J. W. Stansbury, and F. J. Burke, "Self-adhesive resin cements: chemistry, properties and clinical considerations," Journal of Oral Rehabilitation, vol. 38, no. 4, pp. 295-314, 2011.

[22] H. U. V. Gerth, T. Dammaschke, H. Zuchner, and E. Schafer, "Chemical analysis and bonding reaction of RelyX Unicem and Bifix composites- a comparative study," Dental Materials, vol. 22, no. 10, pp. 934-941, 2006.

[23] C. Holderegger, I. Sailer, C. Schumacher, R. Schlapfer, C. Hammerle, and J. Fischer, "Shear bond strength of resin cements to human dentin," Dental Materials, vol. 24, no. 7, pp. 944-950, 2008.

[24] J. Puppin-Ronrni, D. Sundfeld, A. R. Costa et al., "Effect of hydrofluoric acid concentration and etching time on bond strength of lithium disilicate glass ceramic," Operative Dentistry, vol. 42, no. 6, pp. 606-615, 2017.

[25] D. Sundfel Neto, L. Z. Naves, A. R. Costa et al., "The effect of hydrofluoric acid concentration on the bond strength and morphology of the surface and interface of glass ceramics to a resin cement," Operative Dentistry, vol. 40, no. 5, pp. 470479, 2015.

[26] V. K. Kalavacharla, N. C. Lawson, L. C. Ramp, and J. O. Burgess, "Influence of etching protocol and silane treatment with a universal adhesive on lithium disilicate bond strength," Operative Dentistry, vol. 40, no. 4, pp. 372-378, 2015.

[27] J. Brendeke and M. Ozcan, "Effect of physiochemical aging conditions on the composite-composite repair bond strength," Journal of Adhesive Dentistry, vol. 9, no. 4, pp. 399-408, 2007.

[28] C. Cotes, M. Cardoso, R. M. Melo, L. F. Valandro, and M. A. Bottino, "Effect of composite surface treatment and aging on the bond strength between a core build-up composite and a luting agent," Journal of Applied Oral Science, vol. 23, no. 1, pp. 71-78, 2015.

[29] M. Ozcan and G. Pekkan, "Effect of different adhesion strategies on bond strength of resin composite to compositedentin complex," Operative Dentistry, vol. 38, no. 1, pp. 63-72, 2013.

[30] M. Rinastiti, M. Ozcan, W. Siswomihardjo, and H. J. Busscher, "Effect of surface conditioning on repair bond strengths of non-aged and aged microhybrid, nanohybrid, and nanofilled composite resins," Clinical Oral Investigations, vol. 15, no. 5, pp. 625-633, 2011.

[31] K. J. Chun, H. H. Choi, and J. Y. Lee, "Comparison of mechanical property and role between enamel and dentin in the human teeth," Journal of Dental Biomechanics, vol. 5, pp. 1-7, 2014. 


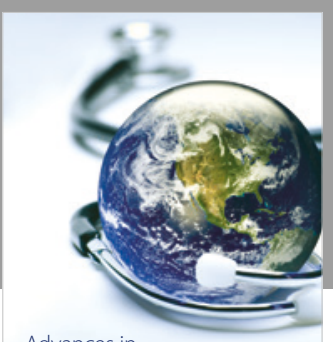

Advances in
Public Health

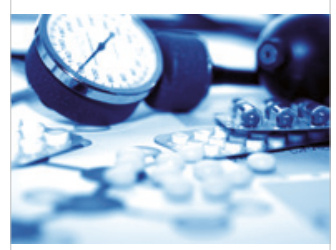

Case Reports in

Medicine

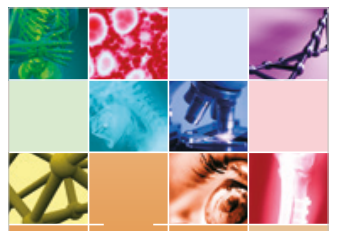

niernational Journal of

Biomaterials
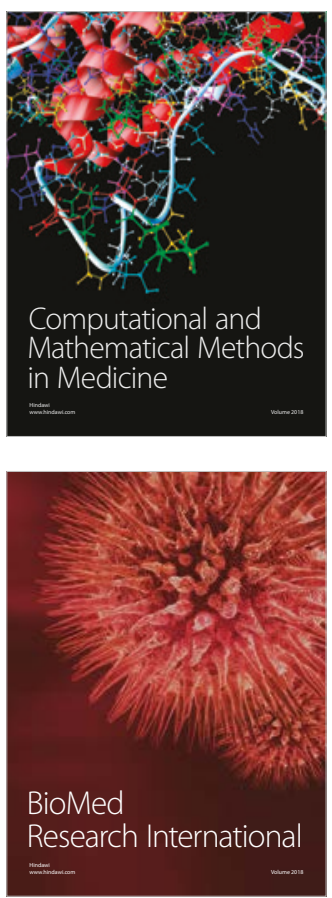

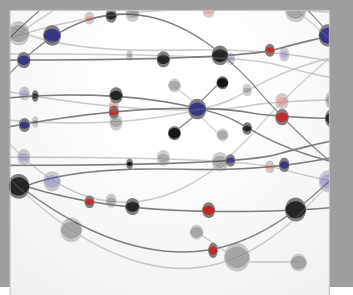

The Scientific World Journal Dentistry

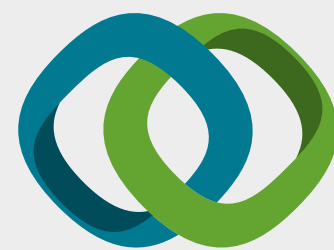

Hindawi

Submit your manuscripts at

www.hindawi.com
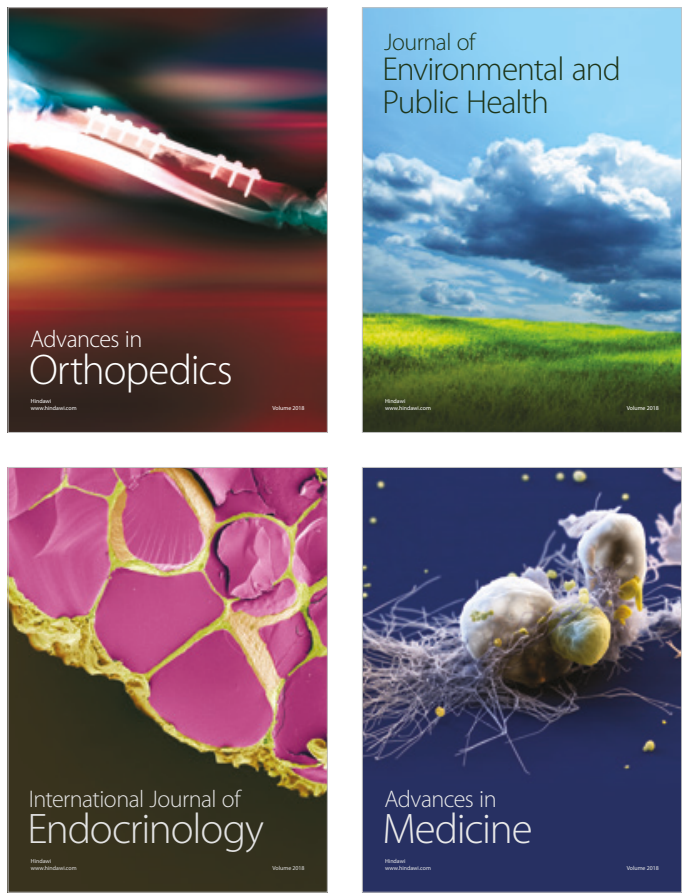
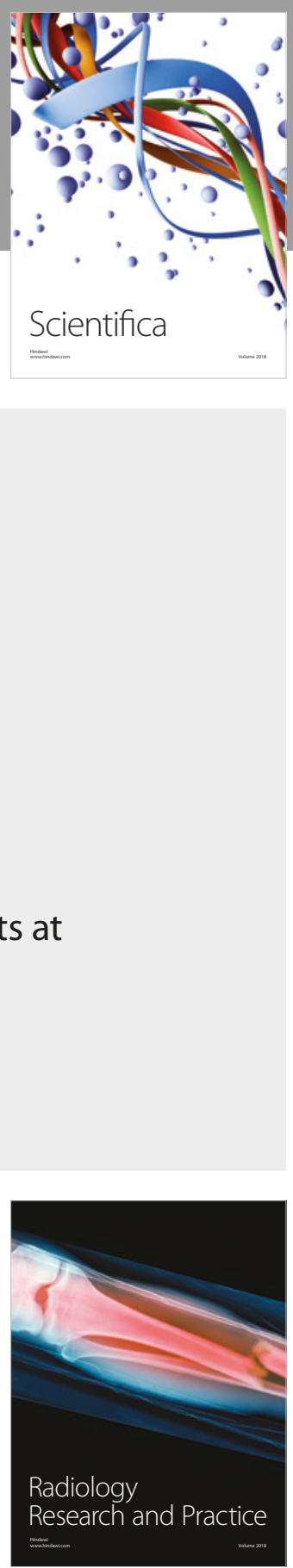

Scientifica

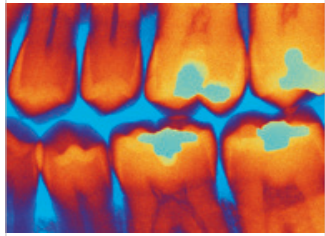

Case Reports in

Dentistry
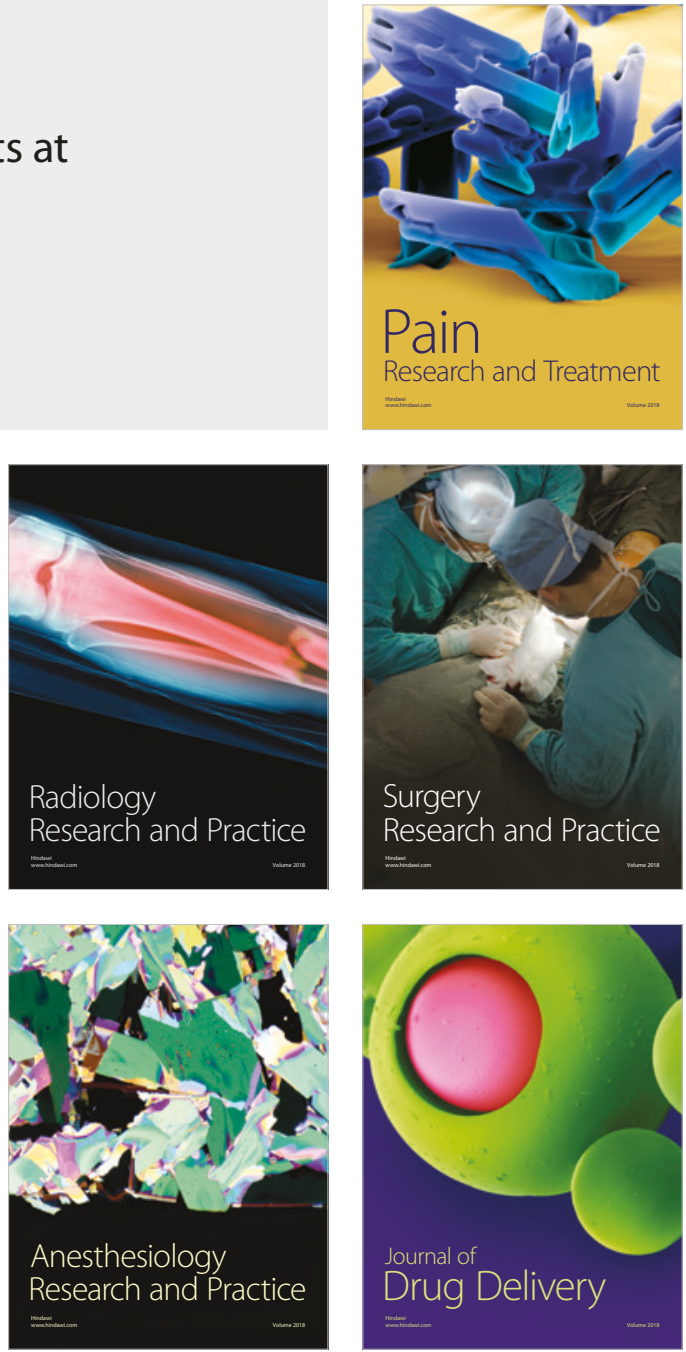